\title{
Filling out the structural map of the NTF2-like superfamily
}

\author{
Ruth Y Eberhardt ${ }^{1,2^{*}}$, Yuanyuan Chang ${ }^{3}$, Alex Bateman ${ }^{2}$, Alexey G Murzin ${ }^{4}$, Herbert L Axelrod ${ }^{5}$, \\ William C Hwang ${ }^{3}$ and L Aravind ${ }^{6}$
}

\begin{abstract}
Background: The NTF2-like superfamily is a versatile group of protein domains sharing a common fold. The sequences of these domains are very diverse and they share no common sequence motif. These domains serve a range of different functions within the proteins in which they are found, including both catalytic and non-catalytic versions. Clues to the function of protein domains belonging to such a diverse superfamily can be gleaned from analysis of the proteins and organisms in which they are found.

Results: Here we describe three protein domains of unknown function found mainly in bacteria: DUF3828, DUF3887 and DUF4878. Structures of representatives of each of these domains: BT_3511 from Bacteroides thetaiotaomicron (strain VPI-5482) [PDB:3KZZ], Cj0202c from Campylobacter jejuni subsp. jejuni serotype O:2 (strain NCTC 11168) [PDB:3K7C], rumgna_01855) and RUMGNA_01855 from Ruminococcus gnavus (strain ATCC 29149) [PDB:4HYZ] have been solved by $X$-ray crystallography. All three domains are similar in structure and all belong to the NTF2-like superfamily. Although the function of these domains remains unknown at present, our analysis enables us to present a hypothesis concerning their role.

Conclusions: Our analysis of these three protein domains suggests a potential non-catalytic ligand-binding role. This may regulate the activities of domains with which they are combined in the same polypeptide or via operonic linkages, such as signaling domains (e.g. serine/threonine protein kinase), peptidoglycan-processing hydrolases (e.g. NlpC/P60 peptidases) or nucleic acid binding domains (e.g. Zn-ribbons).
\end{abstract}

Keywords: NTF2-like superfamily, Protein function prediction, Protein structure, Ligand-binding, JCSG, 3D structure, Protein family

\section{Background}

The NTF2-like superfamily is a large group of related proteins that share a common fold, first observed in the structure of the rat NTF2 (Nuclear Transport Factor 2) protein [1]. It is a versatile fold that can accommodate very different sequences and has no characteristic sequence motif associated with it. The NTF2-like fold has a cone-like shape with a cavity inside and acts as a molecular container that can be adapted to serve a broad range of different functions.

\footnotetext{
*Correspondence: re3@sanger.ac.uk

'Wellcome Trust Sanger Institute, Wellcome Trust Genome Campus, Hinxton, Cambridgeshire, CB10 1SA, UK

${ }^{2}$ European Molecular Biology Laboratory, European Bioinformatics Institute, Hinxton, Cambridgeshire CB10 1SD, UK

Full list of author information is available at the end of the article
}

The NTF2-like proteins can be broadly defined into two functional categories: enzymatically active and nonenzymatically active proteins. The intracellular examples of this fold include most of the enzymatic functions associated with these proteins. These include SnoaL polyketide cyclase, scytalone dehydratase, limonene-1,2epoxide hydrolase and $85-3$-ketosteroid isomerase [2-5]. The extracellular NTF2-like proteins tend to be nonenzymatic and possess small molecule binding activity. Non-enzymatic members of this superfamily include NTF2 [1], a domain found at the C-terminus of calcium/calmodulin dependent protein kinase II which is responsible for the multimerization of these kinases [6] and Mba1, a protein which binds to ribosomes and may function as a receptor [7]. NTF2-like domains have been found in proteins involved in bacterial conjugation where a multiprotein complex, the type IV secretion

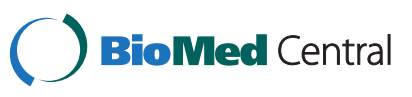


system, mediates transfer of plasmid DNA from a donor to a recipient bacterial cell [8-10]. More recently the non-catalytic NTF2-like domains have also been shown to function as immunity proteins in the bacterial polymorphic toxin systems [11].

Release 27.0 of the Pfam database [12] includes 24 different families as part of the NTF2 superfamily. Of these families, 21 have at least one representative where the three-dimensional structure has been deposited in the PDB. To date, the PDB contains at least 170 structures with NTF2-like fold, including at least 27 structures solved by the Joint Center for Structural Genomics (JCSG). Here we describe the first crystal structures of three Pfam families with NTF2-like folds: DUF3828 [PDB:3KZT] [Pfam:PF12883], DUF 3887 [PDB:4HYZ] [Pfam:PF13026] and DUF4878 [PDB: 3K7C] [Pfam:PF12870].

\section{Results and discussion Domain descriptions}

DUF3828 family [Pfam: PF12883] is annotated in Pfam as a domain of unknown function. It is present in 492 different UniProtKB proteins from 451 different organisms. It is found exclusively in Gram-negative bacteria, with the vast majority of the species it occurs in belonging to the Enterobacteraceae family. [Pfam: PF12870] was previously annotated in Pfam as a lumazine-binding domain, however this has since been found to be incorrect and so we have renamed this family as a domain of unknown function, DUF4878. This domain is present in 650 different UniProtKB proteins from 571 different species. Like DUF3828, DUF4878 is a bacterial family, however it is found in a wider variety of bacterial species. It is found in both Gram-negative bacteria (including Proteobacteria and Bacteroidetes) and Grampositive species (including Firmicutes and Actinobacteria). Finally, DUF3887 family [Pfam: PF13026] is another domain of unknown function. This domain is present in 364 different UniProtKB proteins from 262 different species. It is predominantly found in Firmicutes, but is also present in other phyla, including several Archaeal species.

All three of these domains are of a similar length (around 100 amino acids). The N-terminus of DUF3828 (Figure 1) contains a pair of conserved aromatic amino acids (phenylalanine and tyrosine). There is a conserved aspartic acid in the middle of the domain and close to this is a conserved glutamine. A conserved tryptophan is located near the C-terminus, closely followed by two conserved hydrophobic amino acids. DUF3887 (Figure 2) contains a highly conserved glycine in the middle of the domain and two conserved hydrophobic amino acids near the C-terminus. DUF4878 (Figure 3) contains a conserved glycine about 25 amino acids into the domain and a conserved tryptophan near the $\mathrm{C}$-terminus.

\section{Domain architectures}

In Pfam, 224 of the 492 proteins (45\%) containing DUF3828 also contain a DUF4878 domain at the Cterminus (Figure 4). Given the potential significance of this observation, we performed further investigation into the taxonomic distribution of these proteins. Using EvolView [14] we plotted a species tree containing members of the RP75 set of representative proteomes [15] which possess proteins containing DUF3828 and/or DUF4878 (Figure 5). Surprisingly, we found that only two of the 113 species in this tree possessed both domains, and therefore we conclude that the co-occurrence of these domains is not likely to be significant and is an artifact caused by the sequencing of a disproportionately large number of Escherichia coli strains compared to the other species these domains are found in.

Besides the apparent co-occurrence of DUF3828 and DUF4878, the three DUFs also occur in several other architectures in Pfam. These can be split roughly into three categories: Architectures suggestive of communication with extracellular ligand-sensing or intracellular signaling domains (Figure 4A), solo or multi-domain secreted and lipid-anchored architectures (Figure 4B) and fusions to $\mathrm{C}$-terminal peptidase or other hydrolase domains (Figure 4C).

In the first category (Figure 4A), the intracellular domains to which the extrinsic DUF is linked include protein kinase domains and three distinct versions of zinc ribbons, which could potentially bind nucleic acids. These architectures are comparable to other signaling proteins where extracellular ligand domains are linked to intracellular signaling domains. This category also includes fusions of the DUF with the sodium pump associated oxaloacetate decarboxylase $\gamma$ chain (OAD gamma). In the third category (Figure $4 \mathrm{C}$ ) we observed independent fusions to metallopeptidase (M23), DUF2324 (a transmembrane domain which is a member of the Peptidase U clan), a beta-lactamase and an $\alpha / \beta$ hydrolase domain (Abhydrolase). In all of these cases the DUF is present at the $\mathrm{N}$ terminus and the hydrolase domain at the $\mathrm{C}$-terminus. NTF2-like domains have been observed with $\alpha / \beta$ hydrolases before: both SnoaL-like domain [Pfam:PF12680] and DUF4440 [Pfam:PF14534] co-occur with $\alpha / \beta$ hydrolase domains.

\section{Genomic context}

We studied the genomic context of proteins containing DUF3828, DUF3887 and DUF4878. In doing this we hoped to glean information about the possible function of these domains. As a result we uncovered a conserved association with DUF3828, which in diverse gammaproteobacteria, betaprotebacteria and bacteroidetes is combined in an operon with a gene coding for a protein of the NlpC/P60 superfamily with a papain-like peptidase 
fold (e.g. gi: 489959630 from Enterobacter cloacae) [17]. These domains are known to function as peptidases/amidases in that cleave amide/peptide linkages in the bacterial cell wall. Several of these proteins additionally contain further C-terminal domains such as EF-hands, metallopeptidase family M23 and glycohydrolases of the lysozyme [Pfam:PF00959] or the Chitinase Class I [Pfam: PF00182] families. Thus, domains point to catalytic activities that process both the peptide and glycosidic linkages in peptidoglycan.

\section{Structure description}

The crystal structure of a DUF3828 protein, BT_3511 protein [UniProtKB:Q8A1Z7] from Bacteroides thetaiotaomicron (strain VPI-5482), was determined to $2.1 \AA$ resolution by MAD method and deposited to PDB as [PDB:3KZT]. The final model includes two molecules (residues 26-167), five 1,2-ethanediol, two sulfate ions and 118 water molecules in the asymmetric unit. The structure is mainly composed of three helices, one $3_{10}$ helix and 4 beta strands. Gly0 (that remained at the
$\mathrm{N}$-terminus after cleavage of the expression/purification tag), the region from Lys26 to Pro34 was disordered and not modeled. All the side chains were fully modeled because of the complete electron density. The Matthews coefficient $\left(V_{M}\right)$ is $2.05 \AA^{3} \mathrm{Da}^{-1}$ and the estimated solvent content is $39.97 \%$. The Ramachandran plot produced by MolProbity [18] shows that $96.9 \%$ of the residues are in favored regions, with no outliers.

The crystal structure of a DUF4878 protein, Cj0202c protein [UniProtKB:Q0PBT7] from Campylobacter jejuni subsp. jejuni serotype O:2 (strain NCTC 11168), was determined to $2.0 \AA$ resolution by MAD method and was deposited to PDB as [PDB:3K7C]. The final model includes four molecules (residues 1-113), one chloride ion, thirteen di hydroxyethyl ether (PEG), six triethylene glycol and 134 water molecules in the asymmetric unit. The structure is mainly composed of three helices and four beta strands. Gly0 (which remained at the N-terminus after cleavage of the expression/purification tag), the region from Met1 to Ser5 was disordered and not modeled. All the side chains were fully modeled

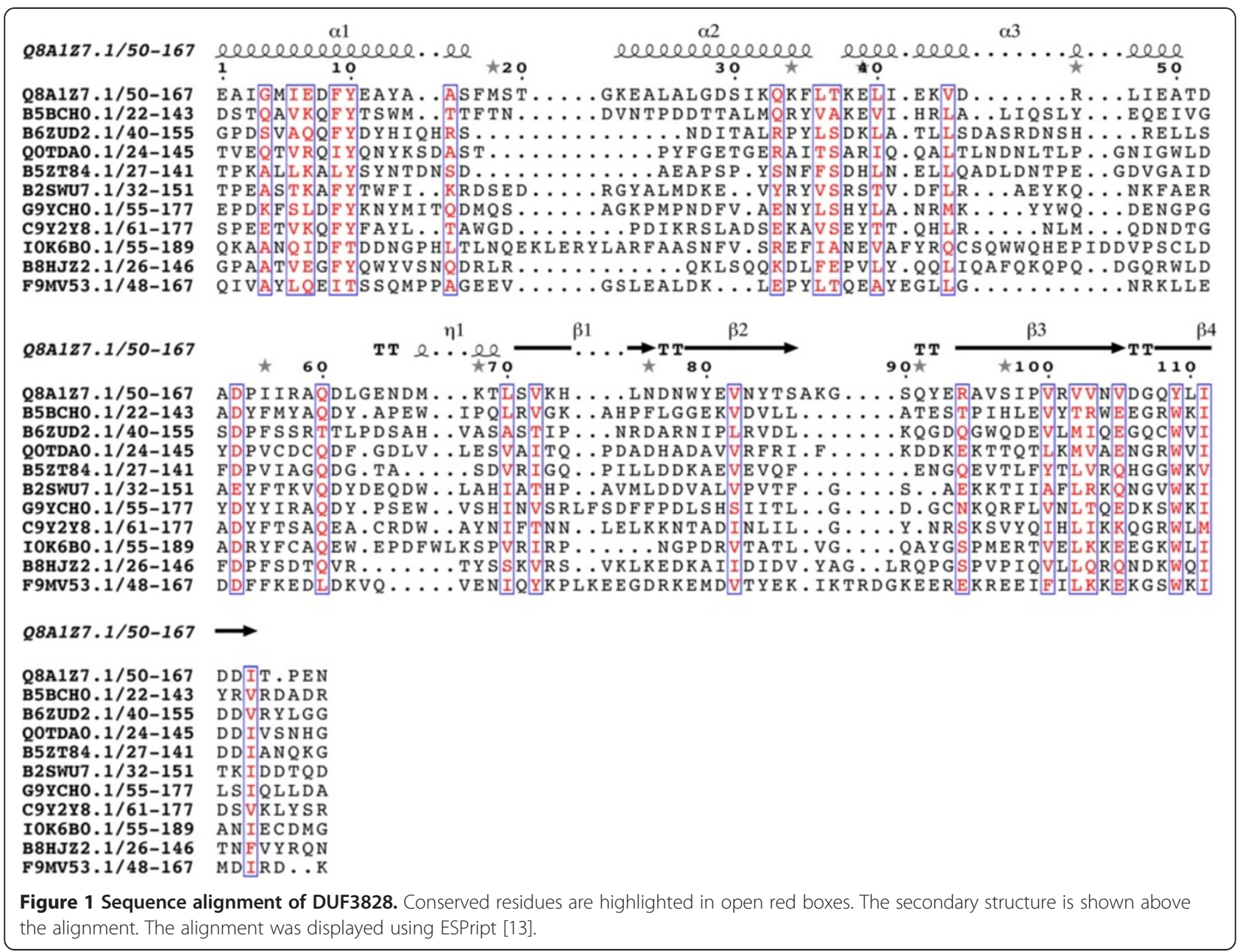




\begin{tabular}{|c|c|c|c|c|}
\hline$A 7 B 2 S 7.1 / 55-147$ & 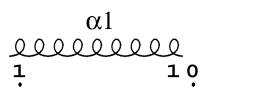 & $\begin{array}{c}\alpha 2 \\
\text { ele.e.e }\end{array}$ & $\stackrel{\alpha 3}{\text { elee }} \star \star$ & $\begin{array}{c}\text { a4 } \\
\text { elebelelel.e } \\
30 \text { ? }\end{array}$ \\
\hline A7B2S7.1/55-147 & AMEDIE I A Q S KD. & YESW. KSRF & TKDLQSSLT. & EESYDSYLKIL. EKQ \\
\hline AOR8Q0.1/36-160 & TEQTEEMKVYRE. & . VHEKYDMKI & $N K E I N K A L Q L W E V A K E K G G K E I$ & TNATYKEDVQKVTTNMLEDI \\
\hline AORFH7.1/37-125 & AEE IVIS L LNEAK. & YKEV.HEKF & DS KMTAALS. & E E KM. KDLTPI I E KA \\
\hline A4AWI0.1/19-104 & AS KF S KYNA SN. & YEDV. HNML & DEVMKS I I P & KE KMTDF L Y TVR SNF \\
\hline A5IK81.1/23-112 & A F L FV Q H L T S EN & . FESA. LNMC. & SNQVKA L S & VQSLSNIWNSLKAQL \\
\hline A7AYH7.1/40-142 & SEA IVIS S F S TP. & . DEV . FDQYE & EMS E LQLDLMLL. & F L S M I EAWKA GEAEC \\
\hline A7VCT $0.1 / 40-133$ & A T Y Y F M M D GD . & . FETF. FNAL. & & AETIQETWEEEVDKL \\
\hline A9KL18.1/74-163 & SAKLTHDLLTEN. & . FEEV.YSYL. & EDTVKE LS & LPDLEKAFHSTVERI \\
\hline A9VKZ2.1/96-188 & TLSFIRHMNS ED. & YKSA. FDLT & SRSLQKIIS & KEW L KS Y WE G L PVQ L \\
\hline B1I2Q6.1/43-137 & $A E N I \amalg Q A F N D D D$. & . YDRF.FKDF. & SQTEKRAAPP & EPEFLETNEQIKNRI \\
\hline B5CV17.1/28-117 & $A Q Q \mathrm{Q} Q \mathrm{QWMKNAQ}$. & . SDSI. YACF. & $D A K M Q Q A V P$ & LSQLNNMWSQMEQQL \\
\hline C8WGP2.1/123-216 & AQ KVA E VVGEGD. & Y DKL. RPML & DDAAAEALT & E PVMNDAHA LF GDDW \\
\hline D5NWE 6.1/118-208 & AES F D D A L T A HQ. & . WENA. AQQL. & GPVI G S L D & AEGLAATWAQVTALG \\
\hline D6E742.1/53-143 & AR D A D L FVARD. & Y Y G L . AALF. & GEPAPA $S$ D & FE KAGRAAEDV \\
\hline E0I2S6.1/137-226 & VD T Y I N G F F S G K. & FEEI.FELQ & $T E Q V K A \mid S L S$ & L K G A Y AN \\
\hline E4LU36.1/46-137 & ALQFVKDLTQRK. & Y Y KL. QKDY & Y D E QMH KA V R & E D S F Q KQ L D A Y N D L \\
\hline E6TQB6.1/58-147 & A E EY I S H L SQGD. & FEIA. YDFF. & . NEVMKA IE & VSELGDIWETLESQV \\
\hline F4XDN8.1/40 & GR S VM E L L N Q GD. & WQAV. YDQL & RSDAKETTS & PENIESYMNSAVEKA \\
\hline F6D290.1/13-102 & $A Q N F V D Q F L Q A D$. & . FAAA. SSRF. & DDQMKVVFP & EVKFQEFWQRFIGPA \\
\hline F7JPH9.1/45-140 & AE C VVVGWFNEQE. & . YGKI. IEAG. & SEELKELYS & EEDIGSEGKDRLKKC \\
\hline G2MPX0.1/64-153 & TQDFLEALIAES. & FDEA.Y Y YA & SPEFAQSVP & QDN L VQQWEAN I I P L \\
\hline HOKRP $6.1 / 25-114$ & A. S D FV D D LFTKK & YTES. VKLE. & D DEV KDKM T & E TVLEMVNGQISGMF \\
\hline H1PIQ7.1/41-134 & $\mathrm{S}$ KN F V H N LNR GD & FDAC. YNSL. & SPDMQD KTG & IERLHALLDP I LGFL \\
\hline H3К 6 P $6.1 /$ & A E T F I S G T T TEK & Y Y KL. VANI & DNGLKKNF & A KA Y D A L KN EVKTKF \\
\hline Q8EMU8.1/26- & $D N L I A G N$ & EDV . & $Q L Q E T Y T$ & KEELQASWKEMTVNE \\
\hline
\end{tabular}

\begin{tabular}{|c|c|c|c|c|c|c|}
\hline \multirow{2}{*}{$A 7 B 2 S 7.1 / 55-147$} & $\beta 2$ & \multicolumn{2}{|l|}{$\beta 3$} & \multicolumn{3}{|l|}{$\beta 4$} \\
\hline & 50 & 70 & & 80 & & \\
\hline 7B2S7.1/55-147 & $\mathrm{K}$ 国F K & DI & & Y S & DEI & $\mathbb{N} \| \mathrm{V}$ \\
\hline R $8 Q 0.1 / 36-160$ & D H I R K E I R V P & & & & & \\
\hline ORFH7.1/37-125 & G T F E I I E KQS & LYTVI L VAKY & $K E Q R T$. & F I T T & $\mathrm{NDKEE}$ & \\
\hline 1 AWT & 1 & & & & & \\
\hline $5 \mathrm{IK} 81$. & S D I & $\mathrm{KF}$ & & A. & & \\
\hline $7 \mathrm{~A}$ & GAFKS Y Y E F & GIVVS & & IEFTF & $D E E Q Q$ & $\mathrm{QD} D$ \\
\hline & $\mathrm{GC}$ & C & & & & \\
\hline $9 \mathrm{KL} 18]$. & $D$ A I S I & $\mathrm{EY}$ & & & $\mathrm{NKDCK}$ & \\
\hline $9 \mathrm{~V}$ & $\mathrm{~A} G \mathrm{Y} F \mathrm{~F} I \mathrm{I} G \mathrm{EV}$ & VF & & $\mathrm{I} \mathrm{KL}$ & D P S G K & $\mathrm{K} I \mathrm{D} D$ \\
\hline & $\mathrm{S}$ & & & & & \\
\hline $5 \mathrm{C}$ & $\mathrm{E} E$ & & & FI & & $\mathrm{K} V \mathrm{~N} \mathrm{NG}$ \\
\hline $\mathrm{c} 8 \mathrm{~W}$ & $\mathrm{~S}$ & MGMT & & $G \mathrm{~F}$ & & $\mathrm{~K}] \mathrm{I}=\mathrm{I}$ \\
\hline D5N & $\mathrm{E} A \mathrm{R}$. & & & & NADG T & $\mathrm{T} / \mathrm{T}$ \\
\hline $6 \mathrm{E}$ & $\mathrm{ESY}$ & $\mathrm{DGV}$ & & & & \\
\hline EOI & $\mathrm{GE}$ & & & & & \\
\hline $\mathrm{E} 4 \mathrm{~L}$ & GS L I $E A$ & & & & & I Q Q C \\
\hline E6T & $\mathrm{GT}$ & & & & & \\
\hline $\mathrm{F} 4 \mathrm{X}$ & $\mathrm{K} E \mathrm{E}$ & $\mathrm{H}$. & & & & $\mathrm{V}=\mathrm{L}$ \\
\hline F6I & $G N L L R L$ & & & & & \\
\hline F7J & GA F R $K I$ & $G E A$ & & $L F$ & & \\
\hline G2MP & GE F N F T S I & GFDVVC & & $\mathrm{FSVQF}$ & & \\
\hline & G D Y K KVV I S & I P M & & $\mathrm{VPF}$ & & $\mathrm{K} V \mathrm{LC}$ \\
\hline-134 & $\mathrm{G}|\mathrm{KF} E \mathrm{E}| \mathrm{F} R \mathrm{R} G \mathrm{~N}$ & STYVC & & $\mathrm{FTII}$ & DE & \\
\hline & SKFYSFERYNN & & & I F & & \\
\hline & S S KQQ & & & & & \\
\hline
\end{tabular}

Figure 2 Sequence alignment of DUF3887. Conserved residues are highlighted in open red boxes. The secondary structure is shown above the alignment. The alignment was displayed using ESPript [13].

because of the complete electron density. The Matthews coefficient $\left(V_{M}\right.$; $)$ is $2.3 \AA^{3} \mathrm{Da}^{-1}$ and the estimated solvent content is $46.46 \%$. The Ramachandran plot produced by MolProbity shows that $97.4 \%$ of the residues are in favoured regions, with no outliers.

The crystal structure of a DUF3887 protein, the hypothetical protein Rumgna_01855 [UniProtKB:A7B2S7] from Ruminococcus gnavus (strain ATCC 29149), was determined to $2.25 \AA$ resolution by MAD method and was deposited to PDB as [PDB:4HYZ]. The final model includes two molecules (residues 36-149), six chloride ions, six sulfate ions, eight glycerol and 107 water molecules in the asymmetric unit. The structure is mainly composed of four helices, four turns, and five beta strands. Only Gly0 (that remained at the N-terminus after cleavage of the expression/purification tag) was disordered and not modeled. All the side chains were fully modeled because of the complete electron density. The Matthews coefficient $\left(V_{M}\right)$ is $3.00 \AA^{3} \mathrm{Da}^{-1}$ and the estimated solvent content is $58.98 \%$. The Ramachandran plot produced by MolProbity shows that $99.6 \%$ of the residues are in favored regions, with no outliers.

Comparison of these three structures showed that they are significantly similar to each other, especially for [PDB:3K7C] and [PDB:4HYZ]. FATCAT results showed that the structures of [PDB:3KZT] and [PDB:4HYZ] are 


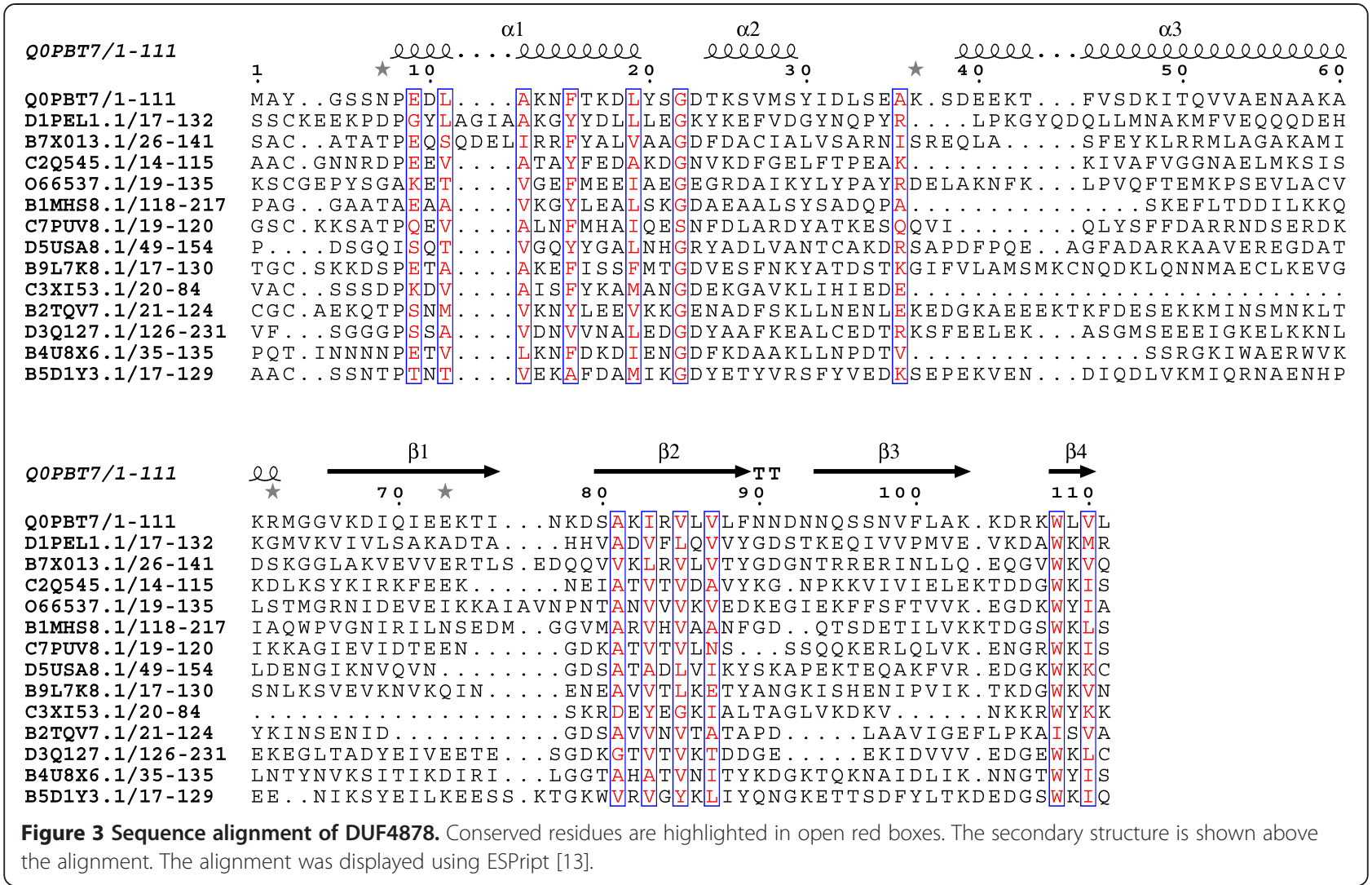

significantly similar with P-value of $1.53 \mathrm{e}^{03}$ and the structure alignment has 85 equivalent positions with an RMSD of $1.72 \AA$; the structures of [PDB:3KZT] and [PDB:3K7C] are significantly similar with P-value of $2.57 \mathrm{e}^{-03}$ and the structure alignment has 95 equivalent positions with an RMSD of $2.53 \AA$; the structures of [PDB:4HYZ] and [PDB:3K7C] are significantly similar with $\mathrm{P}$-value of $1.55 \mathrm{e}^{-}{ }^{06}$ and the structure alignment has 95 equivalent positions with an RMSD of $2.99 \AA$ [19]. In all cases, FATCAT program detected flexibility in the structure, mostly limited to the relative position of helices with respect to the central beta sheet. There is low sequence similarity between the three structures, and no positions are conserved in all three structures.

The three structures ([PDB:3K7C], [PDB:3KZT] and [PDB:4HYZ]) all possess NTF2-like folds, despite being dissimilar in sequence (Figures 6 and 7, Table 1). A hydrophobic cavity with the potential for ligand-binding has been described in NTF2-like proteins before [20]. We used the MarkUs functional annotation server to locate potential cavities within the three structures [21]. All three structures contain predicted cavities, but the position of these cavities is not conserved between the structures. The structure of $[\mathrm{PDB}: 3 \mathrm{~K} 7 \mathrm{C}]$ differs from that of [PDB:3KZT] and [PDB:4HYZ] in that it lacks the edge strands in the beta-sheet but has a longer helix on the opposite side. It has a shallow cavity with positive electrostatic potential that contains a bound PEG molecule in the crystal structure. Notably, in dimers seen in the crystal structure of [PDB:3K7C] the cavities of individual subunits combine in a contiguous groove that can accommodate a larger ligand than a conventional NTFlike fold can. [PDB:4HYZ] has a cavity of a similar size in a similar position with weakly positive electrostatic potential. Sequence conservation in the region of the cavities in [PDB:3K7C] and in [PDB:4HYZ] is poor. In contrast, the cavity found in [PDB:3KZT] has a negative electrostatic potential, this cavity includes two highly conserved aspartic acid residues (D-103 and D-110) which may be of significance.

DALI [24] searches revealed that [PDB:3KZC] and [PDB:4HYZ] are more similar to each other than they are to most other members of the NTF2-like superfamily (Z-score 9.8), however [PDB:3KZT] is more distantly related. [PDB:3KZT] is most similar to [PDB:2UX0] (Z-score 8.8) which contains a Calcium/calmodulin dependent protein kinase II association domain [Pfam: PF08332], also a member of the NTF2-like superfamily. This domain functions as an oligomerisation domain [25]. It is also significantly similar to [PDB:2BHM] (Z-score 8.5), a member of the VirB8 family [Pfam: PF04335], a component of the type IV secretion system [10]. It is significantly less similar to [PDB:3K7C] (maximum Z-score of 6.4 when compared to chain A), and 


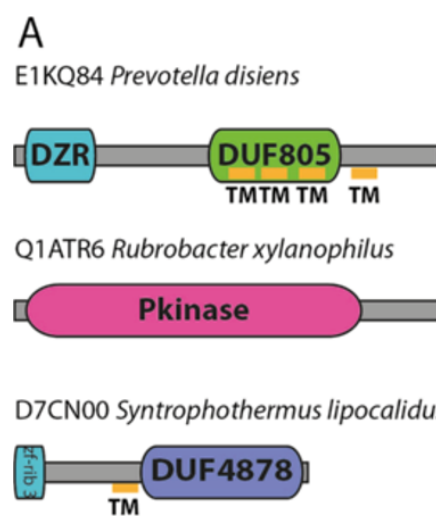

B A8FK03 Campylobacter jejuni SDUF4878 Q31WP1 Shigella boydii

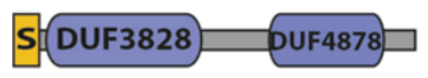

B7JW49 Cyanothece sp strain PCC 8801 S DUF3387 DUF3387
A5KLB2 Ruminococcus torques

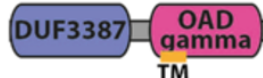

TM

DUF4878

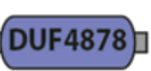

D6L3J5 Parascardovia denticolens

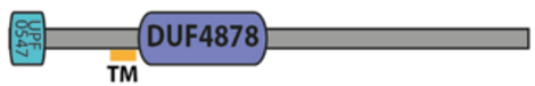

Q8A1Z7 Bacteroides thetaiotaomicron (3KZT)

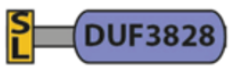

A5MYL5 Clostridium kluyveri

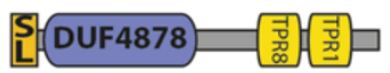

A7B2S7 Ruminococcus gnavus (4HYZ)

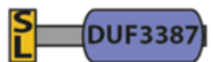

F6B8H2 Desulfotomaculum carboxydivorans

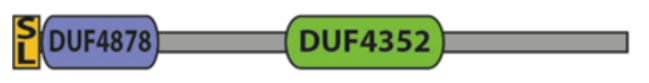

C

FOLJR3 Thermococcus barophilus

A8F713 Thermotoga lettingae
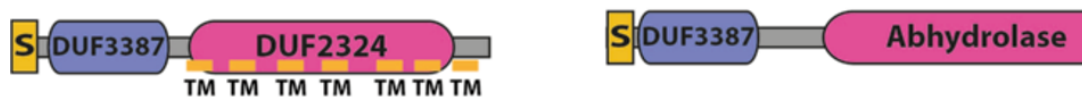

M7MIU3 Formosa sp. AK20

H1ZC72 Myroides odoratus
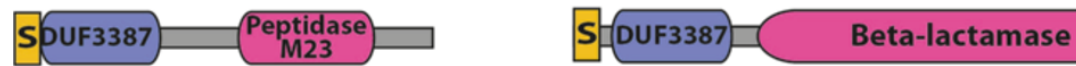

Figure 4 Domain architectures of selected sequences containing NTF2-like domains. Domain architectures were predicted by Pfam [12]. Signal peptides and transmembrane regions were predicted using Phobius [16]. The NTF2-liks DUFs are shown in blue, zinc ribbons in green, TPRs yellow, peptidases and other hydrolases in pink, other DUFs in bright green, and signal peptides (S), lipoboxes (SL) and transmembrane regions (TM) in yellow. Panel $\mathbf{A}$ shows architectures with extracellular ligand-sensing or intracellular signaling domains. Panel $\mathbf{B}$ shows secreted and lipid-anchored architectures. Panel $\mathbf{C}$ shows architectures including hydrolase domains.

[PDB:4HYZ] (maximum Z-score of 6.8 when compared to chain B). [PDB:4HYZ] and [PDB:3K7C] are most similar to members of the SnoAL_3 family [Pfam: PF13474] including [PDB:3GWR] (Z-score 10.1 when compared to [PDB:3K7C]), and the SnoAL_2 family [Pfam:PF12860] including [PDB:3D9R] (Z-score 9.5 when compared to [PDB:3K7C]).

\section{Potential function}

NTF2-like domains include both catalytic and noncatalytic versions that tend to bind small molecules using a common substrate-binding pocket. Our analysis of these DUFs did not reveal conserved polar residues suggestive of catalytic activity in DUF3887 or DUF4878. DUF3828 contains a conserved aspartic acid, which 


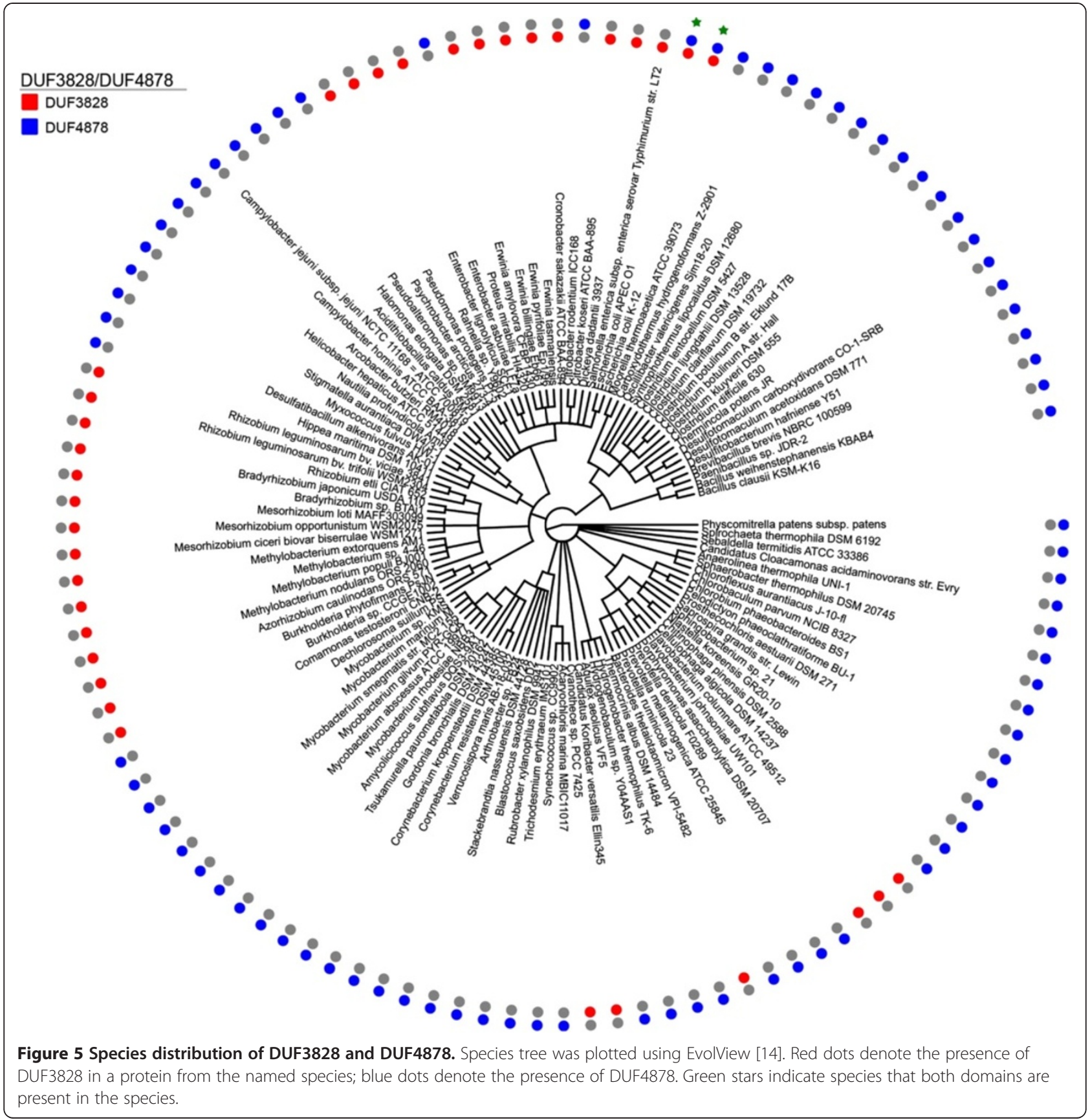

could point to a catalytic function. NTF2-like domains which are enzymatic tend to occur in an intracellular context, however prediction of subcellular localization using Phobius [16] revealed the consistent presence of either N-terminal secretory signals or lipoboxes with a conserved cysteine which helps anchor the protein to the membrane. Those proteins that lack either of these features have transmembrane regions with predicted membrane topologies suggestive of an extracellular location for the DUF (Figure 4). Together these observations suggested that these three DUFs are novel NTF2like domains that are likely to be extracellular domains that recognize a small molecule ligand via their binding pocket.

Further evidence for such a function is offered by the domain architectures of these proteins (Figure 4). Where the DUF is found at the N-terminus of OAD $\gamma$ chain domain the sensing of a ligand could help allosterically 


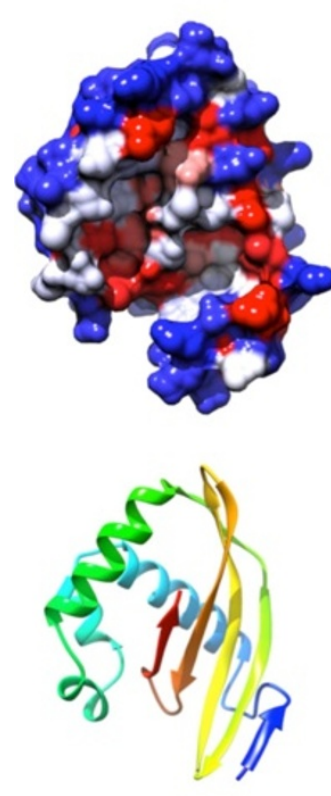

$4 \mathrm{HYZ}$
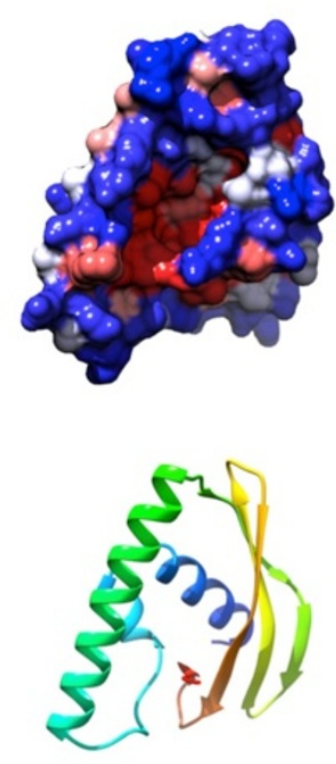

3K7C
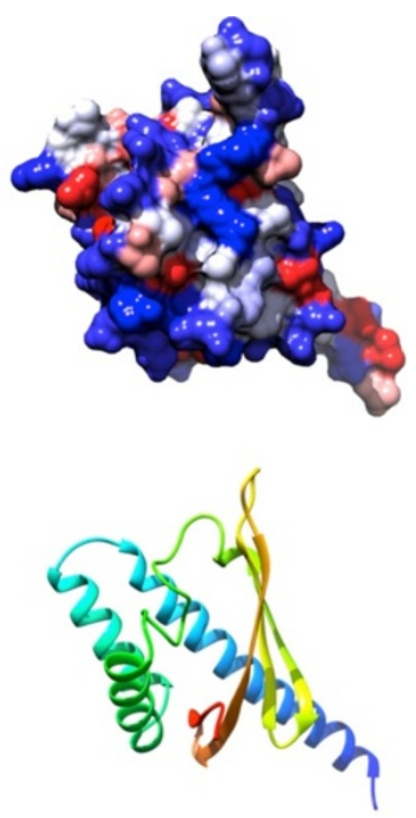

3KZT

Figure 6 Structures of representatives of DUF3828, DUF3887, and DUF4878. [PDB:4HYZ] is a member of DUF3887, [PDB:3K7C] is a member of DUF4878 and [PDB:3KZT] is a member of DUF3828. Structures were aligned with POSA [22] and hydrophobic surface plots generated in Chimera [23].

regulate sodium flux [26]. Where the DUF is found at the $\mathrm{N}$-terminus of a protein containing a C-terminal peptidase or other hydrolase domain, it is conceivable that the sensing of a ligand by the $\mathrm{N}$-terminal DUF regulates the catalytic domain. Similar domain architecture associations were also observed for DUF4352,

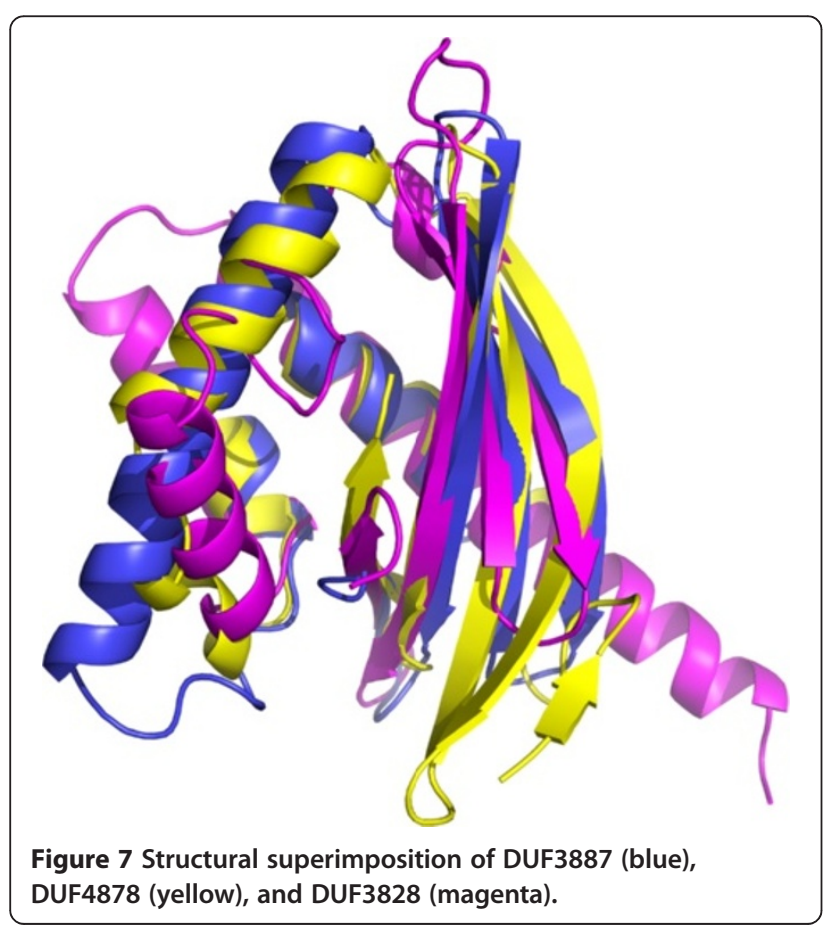

which occurs fused to DUF4878 in certain contexts: DUF4352 is also linked to metallopeptidase (M56), protein kinase and TPR repeats and is also associated with lipid attachment signal or signal peptides or transmembrane regions. Hence it is possible that the two domains perform comparable functions and cooperate in recognition of extracellular ligands on occasions. The versions combined in operons with the $\mathrm{NlpC/P60} \mathrm{like} \mathrm{peptidases/amidases} \mathrm{might} \mathrm{potentially}$ regulate the export and/or the activity of these peptidoglycan hydrolyzing proteins that could have a potentially suicidal effect on the cell. Thus, they could play a role in regulating peptidoglycan remodeling.

\section{Conclusions}

Here we present a comparison of first crystal structures of three DUFs belonging to the NTF2-like superfamily. This work expands our structural knowledge of the

Table 1 Percentage identity of the three proteins for which structure has been determined, calculated using DALI [24]

\begin{tabular}{llll}
\hline PDB ID & 3KZT & 3K7C & 4HYZ \\
\hline 3KZT & & $11 \%$ & $14 \%$ \\
3K7C & $11 \%$ & & $10 \%$ \\
$\mathbf{4 H Y Z}$ & $14 \%$ & $10 \%$ & \\
\hline
\end{tabular}


sequence diverse NTF2 superfamily. Analysis of the three-dimensional structure, sequence and associated domains can provide clues about the likely function of a protein domain. We present a detailed analysis of these three domains, which suggests that they may play a role in binding to small molecule ligands.

\section{Methods}

\section{Sequence and gene context analysis}

Data for families DUF3828 and DUF4878 are taken from Pfam release 27.0 [12]. The definition of DUF3887 has been improved during the course of this work and the updated version will form a part of Pfam release 28.0. Signal peptides and transmembrane domains were predicted using Phobius [16]. A phylogenetic tree was constructed from proteomes in representative proteomes RP75 [15] using the NCBI taxonomy common tree [27]. This was annotated and displayed using EvolView [14].

With the DUF genes as anchors, the gene neighbourhood was also comprehensively analyzed using a custom Perl script. This script uses either the PTT file (downloadable from the NCBI ftp site) or the Genbank file in the case of whole genome shot gun sequences to extract the neighbors of a given query gene. The protein sequences of all neighbors were clustered using the BLASTCLUST program (ftp://ftp.ncbi.nih.gov/blast/documents/blastclust.html) to identify related sequences in gene neighbourhoods. Each cluster of homologous proteins were then assigned an annotation based on the domain architecture or conserved shared domain which were detected using Pfam models and in-house profiles run using RPS-BLAST [28]. This allowed an initial annotation of gene neighbourhoods and their grouping based on conservation of neighborhood associations. In further analysis care was taken to ensure that genes are unidirectional on the same strand of DNA and shared a putative common promoter to be counted as a single operon. If they were head to head on opposite strands they were examined for potential bidirection promoter sharing patterns.

\section{Structure determination}

Protein purification and crystallization was performed by the JCSG crystallomics core [29-31]. All X-ray diffraction data were collected at the Stanford Synchrotron Radiation Lightsource (SSRL) on beamline 11-1. Data sets were collected at $100 \mathrm{~K}$ using a Rayonix MX-325 CCD detector. X-ray diffraction data were collected from a single crystal at wavelengths corresponding to the inflection $\left(\lambda_{1}\right)$, high energy remote $\left(\lambda_{2}\right)$, and peak $\left(\lambda_{3}\right)$ [PDB:3K7C]; the peak $\left(\lambda_{1}\right)$, inflection $\left(\lambda_{2}\right)$, and high energy remote $\left(\lambda_{3}\right)$ [PDB: 4HYZ]; or the inflection $\left(\lambda_{1}\right)$ and high energy remote $\left(\lambda_{2}\right)$ [PDB:3KZT], of a multi-wavelength or a two-wavelength selenium multi- wavelength anomalous diffraction (MAD). The data were integrated and scaled using the XDS and XSCALE programs respectively [32,33] [PDB:3K7C] or the MOSFLM [34] and SCALA [35] programs [PDB:3KZT][PDB:4HYZ]. Data statistics are summarized in Additional file 1: Tables S1-S3. The selenium substructures for the three proteins were solved with SHELXD [36] and the MAD phases were refined with autoSHARP [37]. Iterative automated model building was performed with RESOLVE [38] at a resolution of $2.00 \AA$ [PDB:3K7C] or Arp/Warp [39] at a resolution of $2.15 \AA$ [PDB:3KZT] or with Buccaneer [40,41] at a resolution of $2.25 \AA$ [PDB:4HYZ] from densitymodified electron density. Model completion was performed using the interactive computer-graphics program COOT [42] and MAD-phase-restrained refinement was accomplished using the program REFMAC ver 5.5.0102 [PDB:3K7C], ver 5.5.0053 [PDB:3KZT] [43] or BUSTER ver 2.10.0 [44] [PDB:4HYZ].

\section{Structure validation and deposition}

The quality of the crystal structure was analyzed using the JCSG Quality Control Server [45]. This server verifies: the stereochemical quality of the model using AutoDepInputTool, MolProbity and WHATIF 5.0 [18,46,47]; agreement between the atomic model and the data using SFcheck 4.0 and RESOLVE $[38,48]$; the protein sequence using CLUSTALW [49]; atom occupancies using MOLEMAN2.0 [50]; and consistency of NCS pairs. It also evaluates differences in Rcryst/ Rfree, expected Rfree/Rcryst, and maximum/minimum $\mathrm{B}$-values by parsing the refinement log-file and PDB header. Protein quaternary structure analysis used the EBI PISA server [51]. Atomic coordinates and experimental structure factors have been deposited in the $\mathrm{PDB}$ and are accessible under the codes [PDB:3KZT], [PDB:3K7C] and [PDB:4HYZ]. Electrostatic potential and cavity prediction was performed using the MarkUs functional annotation server [21].

\section{Availabilty of supporting data}

The data sets supporting the results of this article are included within the article (and its Additional file 1: Tables S1-S3).

\section{Additional file}

Additional file 1: Table S1. Data collection and refinement statistic (PDB 3kzt). Table S2. Data collection and refinement statistics (PDB 3k7C). Table S3. Data collection and refinement statistics (PDB 4hyz)

Competing interests

The authors declare that they have no competing interests. 


\section{Authors' contributions}

RYE wrote the majority of the manuscript and produced Figures 1, 2, 3, 4 and 5. YC wrote a section of the manuscript and produced Figure 7. AB contributed to the study ideas and organization of the manuscript. AGM contributed to structure descriptions. HLA wrote structure determination and validation methods. WCH provided Figure 6. LA provided potential function discussion. All authors read and approved the final manuscript.

\section{Acknowledgements}

We are grateful to the Sanford Burnham Medical Research Institute for hosting the DUF annotation jamboree in June 2013, which allowed the authors to collaborate on this work. We would like to thank all the participants of this workshop for their intellectual contributions to this work who, in addition to the authors, were Penny Coggill, Debanu Das, Robert D. Finn, Adam Godzik, Lucasz Jaroszewski, Padmaja Natarajan, Marco Punta, Neil Rawlings, Daniel Rigden, Mayya Sedova, Anna Sheydina and John Wooley. We thank the members of the JCSG high-throughput structural biology pipeline for their contribution to this work.

\section{Funding}

Wellcome Trust (grant numbers WT077044/Z/05/Z); Howard Hughes Medical Institute; Work by LA is supported by the intramural funds of the National Library of Medicine, USA.; NIH (R01GM101457); Work by AGM was supported by the UK Medical Research Council [MC_U105192716]; This work was supported in part by National Institutes of Health Grant U54 GM094586 from the NIGMS Protein Structure Initiative to the Joint Center for Structural Genomics. The DUF annotation jamboree was supported by National Science Foundation (IIS-0646708 and IIS-1153617); Portions of this research were carried out at the Stanford Synchrotron Radiation Lightsource, a Directorate of SLAC National Accelerator Laboratory and an Office of Science User Facility operated for the U.S. Department of Energy Office of Science by Stanford University. The SSRL Structural Molecular Biology Program is supported by the DOE Office of Biological and Environmental Research, and by the National Institutes of Health, National Institute of General Medical Sciences (including P41GM103393). The contents of this publication are solely the responsibility of the authors and do not necessarily represent the official views of NIGMS, NCRR or NIH.

\section{Author details}

${ }^{1}$ Wellcome Trust Sanger Institute, Wellcome Trust Genome Campus, Hinxton, Cambridgeshire, CB10 1SA, UK. '2European Molecular Biology Laboratory, European Bioinformatics Institute, Hinxton, Cambridgeshire CB10 1SD, UK. ${ }^{3}$ Sanford Burnham Medical Research Institute, 10901 North Torrey Pines Road, La Jolla, CA 92037, USA. ${ }^{4}$ MRC Laboratory of Molecular Biology, Francis Crick Avenue, Cambridge Biomedical Campus, Cambridge CB2 0QH, UK. ${ }^{5}$ Stanford Synchrotron Radiation Lightsource, SLAC National Accelerator Laboratory, Menlo Park, CA, USA. ${ }^{6}$ National Center for Biotechnology Information, NLM, NIH, Bethesda, MD 20814, USA

Received: 11 July 2013 Accepted: 15 November 2013

Published: 19 November 2013

\section{References}

1. Bullock TL, Clarkson WD, Kent HM, Stewart M: The 1.6 angstroms resolution crystal structure of nuclear transport factor 2 (NTF2). J Mol Biol 1996, 260(3):422-431

2. Sultana A, Kallio P, Jansson A, Wang JS, Niemi J, Mantsala P, Schneider G: Structure of the polyketide cyclase SnoaL reveals a novel mechanism for enzymatic aldol condensation. EMBO J 2004, 23(9):1911-1921.

3. Nakasako M, Motoyama T, Kurahashi Y, Yamaguchi I: Cryogenic X-ray crystal structure analysis for the complex of scytalone dehydratase of a rice blast fungus and its tight-binding inhibitor, carpropamid: the structural basis of tight-binding inhibition. Biochemistry 1998, 37(28):9931-9939.

4. Arand M, Hallberg BM, Zou J, Bergfors T, Oesch F, van der Werf MJ, De Bont JA, Jones TA, Mowbray SL: Structure of Rhodococcus erythropolis limonene-1,2-epoxide hydrolase reveals a novel active site. EMBO J 2003, 22(11):2583-2592.

5. Kim SW, Cha SS, Cho HS, Kim JS, Ha NC, Cho MJ, Joo S, Kim KK, Choi KY, Oh $\mathrm{BH}$ : High-resolution crystal structures of delta5-3-ketosteroid isomerase with and without a reaction intermediate analogue. Biochemistry 1997, 36(46):14030-14036.
6. Griffith LC, Lu CS, Sun XX: CaMKII, an enzyme on the move: regulation of temporospatial localization. Mol Interv 2003, 3(7):386-403.

7. Ott M, Prestele M, Bauerschmitt H, Funes S, Bonnefoy N, Herrmann JM Mba1, a membrane-associated ribosome receptor in mitochondria. EMBO J 2006, 25(8):1603-1610.

8. Goessweiner-Mohr N, Grumet L, Arends K, Pavkov-Keller T, Gruber CC, Gruber K, Birner-Gruenberger R, Kropec-Huebner A, Huebner J, Grohmann E, et al: The 2.5 A structure of the enterococcus conjugation protein TraM resembles VirB8 type IV secretion proteins. J Biol Chem 2013, 288(3):2018-2028.

9. Porter CJ, Bantwal R, Bannam TL, Rosado CJ, Pearce MC, Adams V, Lyras D, Whisstock JC, Rood Jl: The conjugation protein TcpC from Clostridium perfringens is structurally related to the type IV secretion system protein VirB8 from Gram-negative bacteria. Mol Microbiol 2012, 83(2):275-288.

10. Terradot L, Bayliss R, Oomen C, Leonard GA, Baron C, Waksman G: Structures of two core subunits of the bacterial type IV secretion system, VirB8 from Brucella suis and ComB10 from Helicobacter pylori. Proc Natl Acad Sci U S A 2005, 102(12):4596-4601.

11. Zhang D, De Souza RF, Anantharaman V, lyer LM, Aravind L: Polymorphic toxin systems: Comprehensive characterization of trafficking modes, processing, mechanisms of action, immunity and ecology using comparative genomics. Biol Direct 2012, 7:18.

12. Punta M, Coggill PC, Eberhardt RY, Mistry J, Tate J, Boursnell C, Pang N, Forslund K, Ceric G, Clements J, et al: The Pfam protein families database. Nucleic Acids Res 2012, 40:D290-D301.

13. Gouet P, Robert $X$, Courcelle E: ESPript/ENDscript: Extracting and rendering sequence and $3 \mathrm{D}$ information from atomic structures of proteins. Nucleic Acids Res 2003, 31(13):3320-3323.

14. Zhang H, Gao S, Lercher MJ, Hu S, Chen WH: EvolView, an online tool for visualizing, annotating and managing phylogenetic trees. Nucleic Acids Res 2012, 40:W569-W572.

15. Chen C, Natale DA, Finn RD, Huang H, Zhang J, Wu CH, Mazumder R: Representative proteomes: a stable, scalable and unbiased proteome set for sequence analysis and functional annotation. PloS one 2011, 6(4):e18910.

16. Kall $L$, Krogh A, Sonnhammer EL: A combined transmembrane topology and signal peptide prediction method. J Mol Biol 2004, 338(5):1027-1036.

17. Anantharaman $V$, Aravind L: Evolutionary history, structural features and biochemical diversity of the NIpC/P60 superfamily of enzymes. Genome Biol 2003, 4(2):R11.

18. Chen VB, Arendall WB 3rd, Headd JJ, Keedy DA, Immormino RM, Kapral GJ, Murray LW, Richardson JS, Richardson DC: MolProbity: all-atom structure validation for macromolecular crystallography. Acta Crystallogr D Biol Crystallogr 2010, 66(Pt 1):12-21.

19. Ye Y, Godzik A: Flexible structure alignment by chaining aligned fragment pairs allowing twists. Bioinformatics 2003, 19(Suppl 2):ii246-ii255.

20. Nagata Y, Mori K, Takagi M, Murzin AG, Damborsky J: Identification of protein fold and catalytic residues of gamma-hexachlorocyclohexane dehydrochlorinase LinA. Proteins 2001, 45(4):471-477.

21. Petrey $D$, Fischer $M$, Honig B: Structural relationships among proteins with different global topologies and their implications for function annotation strategies. Proc Natl Acad Sci USA 2009, 106(41):17377-17382.

22. Ye Y, Godzik A: Multiple flexible structure alignment using partial order graphs. Bioinformatics 2005, 21(10):2362-2369.

23. Pettersen EF, Goddard TD, Huang CC, Couch GS, Greenblatt DM, Meng EC, Ferrin TE: UCSF Chimera-a visualization system for exploratory research and analysis. J Comput Chem 2004, 25(13):1605-1612.

24. Holm L, Rosenstrom P: Dali server: conservation mapping in 3D. Nucleic Acids Res 2010, 38:W545-W549.

25. Rellos P, Pike AC, Niesen FH, Salah E, Lee WH, Von Delft F, Knapp S: Structure of the CaMKIldelta/calmodulin complex reveals the molecular mechanism of CaMKII kinase activation. PLOS biology 2010, 8(7):e1000426.

26. Dimroth $P$, Jockel $P$, Schmid M: Coupling mechanism of the oxaloacetate decarboxylase $\mathrm{Na}(+)$ pump. Biochim Biophys Acta 2001, 1505(1):1-14.

27. Sayers EW, Barrett T, Benson DA, Bryant SH, Canese K, Chetvernin V, Church DM, DiCuccio M, Edgar R, Federhen S, et al: Database resources of the national center for biotechnology information. Nucleic Acids Res 2009, 37:D5-D15.

28. Schaffer AA, Wolf YI, Ponting CP, Koonin EV, Aravind L, Altschul SF: IMPALA: matching a protein sequence against a collection of PSI-BLASTconstructed position-specific score matrices. Bioinformatics 1999, 15(12):1000-1011. 
29. Klock HE, Koesema EJ, Knuth MW, Lesley SA: Combining the polymerase incomplete primer extension method for cloning and mutagenesis with microscreening to accelerate structural genomics efforts. Proteins 2008, 71(2):982-994

30. Lesley SA, Kuhn P, Godzik A, Deacon AM, Mathews I, Kreusch A, Spraggon G, Klock HE, McMullan D, Shin T, et al: Structural genomics of the Thermotoga maritima proteome implemented in a high-throughput structure determination pipeline. Proc Natl Acad Sci U S A 2002, 99(18):11664-11669.

31. Elsliger MA, Deacon AM, Godzik A, Lesley SA, Wooley J, Wuthrich K, Wilson IA: The JCSG high-throughput structural biology pipeline. Acta Crystallogr Sect F Struct Biol Cryst Commun 2010, 66(Pt 10):1137-1142.

32. Kabsch W: Automatic processing of rotation diffraction data from crystals of initially unknown symmetry and cell constants. J Appl Cryst 1993, 26:795-800.

33. Kabsch W: Xds. Acta Crystallogr D Biol Crystallogr 2010, 66(2):125-132.

34. Leslie AGW, Powell HR: Processing diffraction data with MOSFLM. In Evolving Methods for Macromolecular Crystallography. Edited by Read RJ, Sussman JL. Dordrech, The Netherlands: Springer; 2007:41-51. volume 245. ISBN 978-1-4020-6314-5.

35. Evans P: Scaling and assessment of data quality. Acta Crystallogr D Biol Crystallogr 2006, 62(Pt 1):72-82.

36. Schneider TR, Sheldrick GM: Substructure solution with SHELXD. Acta Crystallogr D Biol Crystallogr 2002, 58(Pt 10 Pt 2):1772-1779.

37. Vonrhein C, Blanc E, Roversi P, Bricogne G: Automated structure solution with autoSHARP. Methods Mol Biol 2007, 364:215-230.

38. Terwilliger TC: Automated main-chain model building by template matching and iterative fragment extension. Acta Crystallogr D Biol Crystallogr 2003, 59(Pt 1):38-44.

39. Langer G, Cohen SX, Lamzin VS, Perrakis A: Automated macromolecular model building for X-ray crystallography using ARP/wARP version 7. Nature protocols 2008, 3(7):1171-1179.

40. Cowtan K: The Buccaneer software for automated model building. 1. Tracing protein chains. Acta Crystallogr D Biol Crystallogr 2006, 62(9):1002-1011.

41. Cowtan K: Completion of autobuilt protein models using a database of protein fragments. Acta Crystallogr D Biol Crystallogr 2012, 68(Pt 4):328-335.

42. Emsley P, Cowtan K: Coot: model-building tools for molecular graphics. Acta Crystallogr D Biol Crystallogr 2004, 60(Pt 12 Pt 1):2126-2132.

43. Winn MD, Isupov MN, Murshudov GN: Use of TLS parameters to model anisotropic displacements in macromolecular refinement. Acta Crystallogr D Biol Crystallogr 2001, 57(Pt 1):122-133.

44. Smart OS, Womack TO, Flensburg C, Keller P, Paciorek W, Sharff A, Vonrhein $C$, Bricogne G: Exploiting structure similarity in refinement: automated NCS and target-structure restraints in BUSTER. Acta Crystallogr D Biol Crystallogr 2012, 68(Pt 4):368-380

45. Pham L, Christadore L, Schaus S, Kolaczyk ED: Network-based prediction for sources of transcriptional dysregulation using latent pathway identification analysis. Proc Natl Acad Sci U S A 2011, 108(32):13347-13352.

46. Yang H, Guranovic V, Dutta S, Feng Z, Berman HM, Westbrook JD: Automated and accurate deposition of structures solved by X-ray diffraction to the protein data bank. Acta Crystallogr D Biol Crystallogr 2004, 60(Pt 10):1833-1839.

47. Vriend G: WHAT IF: a molecular modeling and drug design program. J Mol Graph 1990, 8(1):52-56.

48. Vaguine AA, Richelle J, Wodak SJ: SFCHECK: a unified set of procedures for evaluating the quality of macromolecular structure-factor data and their agreement with the atomic model. Acta Crystallogr D Biol Crystallogr 1999, 55(Pt 1):191-205.

49. Chenna R, Sugawara H, Koike T, Lopez R, Gibson TJ, Higgins DG, Thompson JD: Multiple sequence alignment with the Clustal series of programs. Nucleic Acids Res 2003, 31(13):3497-3500.

50. Kleywegt GJ: Validation of protein models from Calpha coordinates alone. J Mol Biol 1997, 273(2):371-376.

51. Krissinel E, Henrick K: Inference of macromolecular assemblies from crystalline state. J Mol Biol 2007, 372(3):774-797.

\section{Submit your next manuscript to BioMed Central and take full advantage of:}

- Convenient online submission

- Thorough peer review

- No space constraints or color figure charges

- Immediate publication on acceptance

- Inclusion in PubMed, CAS, Scopus and Google Scholar

- Research which is freely available for redistribution 